\title{
Estilos parentais e comportamento desviante: papel mediador do consumo de álcool em estudantes universitários
}

\author{
Catarina Pinheiro Mota ${ }^{a b *}$, Sandra de Assunção ${ }^{a}$ \\ a Universidade de Trás-os-Montes e Alto Douro - UTAD, Vila Real, Portugal \\ ${ }^{b}$ Centro de Psicologia da Universidade do Porto, Porto, Portugal
}

Recebido em 26 de fevereiro 2020; aceite em 21 de junho de 2020

\author{
PALAVRAS-CHAVE \\ Estilos parentais, \\ risco de consumo \\ de álcool, \\ comportamento desviante, \\ jovens universitários
}

\begin{abstract}
Resumo As relações existentes com as figuras parentais revelam-se importantes para os jovens adultos no que diz respeito ao desenvolvimento de estratégias de coping adequadas e de competências sociais. A presença de um estilo parental democrático parece minimizar o envolvimento dos filhos em comportamentos de risco, nomeadamente o consumo de álcool, tabaco e outras drogas. 0 presente estudo tem como objetivo analisar o papel dos estilos parentais no risco de consumo de álcool e no desenvolvimento de outros comportamentos adictivos e autodestrutivos em estudantes universitários. A amostra foi constituída por 1044 jovens, dos quais 277 (26.5\%) do sexo masculino e 767 (73.5\%) do sexo feminino. Todos os participantes tinham idades compreendias entre os 18 e 25 anos $(M=19.78 ; D P=1.68)$. Recorreu-se a um questionário sociodemográfico e a instrumentos de autorrelato, nomeadamente o Parenting Styles \& Dimensions Questionnaire: Short Version (PSDQ), o Alcohol Use Disorders Identification Test (AUDIT) e a Escala de Comportamentos Desviantes (ECD). Os resultados apontam para um efeito preditor dos estilos parentais negativos (autoritário e permissivo) face ao comportamento adictivo e autodestrutivo dos jovens. Verificou-se ainda, o papel mediador do consumo de álcool na associação entre os estilos parentais negativos e o comportamento adictivo e autodestrutivo.
\end{abstract}

( 2020 Fundación Universitaria Konrad Lorenz. Este é um artigo Open Access distribuído sob os termos da Licença Creative Commons CC BY-NC-ND (http://creativecommons.org/licenses/by-ncnd/4.0/).

Parenting styles and deviant behavior: Mediating role of alcohol consumption among university students

Abstract The relationships with parental figures are important for young adults in the developing of appropriated coping strategies and social skills. The presence of a democratic parenting style seems to minimize the involvement of the children in risk behaviors, namely the consumption of alcohol, tobacco and other drugs. The present study aims to analyze the role of parenting styles in the risk of alcohol consumption, as also the development of

* Autor para correspondência:

Correio eletrónico: catppmota@utad.pt

https://doi.org/10.14349/sumapsi.2020.v27.n2.4

ISSN 0121-4381, ISSN-E 2145-9797/@ 2020 Fundación Universitaria Konrad Lorenz. This is an open access article under the CC BY-NC-ND license (http://creativecommons.org/licenses/by-nc-nd/4.0/). 
additive and self-destructive behavior in university students. The sample consisted of 1044 young people, of whom 277 (26.5\%) were male and 767 (73.5\%) were female. All participants were between 18 and 25 years of old $(M=19.78$; $S D=1.68)$. Were used a sociodemographic questionnaire and self-report instruments, namely the Parenting Styles \& Dimensions Questionnaire: Short Version (PSDQ), the Alcohol Use Disorders Identification Test (AUDIT) and the Deviant Behavior Scale (ECD). The results point to a predictive effect of negative parenting styles (authoritarian and permissive) in relation to the additive and self-destructive behavior of the university students. The mediating role of alcohol consumption in the association between negative parenting styles and additive and self-destructive behavior was also verified.

(c) 2020 Fundación Universitaria Konrad Lorenz. This is an open access article under the CC BYNC-ND license (http://creativecommons.org/licenses/by-nc-nd/4.0/).

Os estilos parentais são definidos como sendo a constelação de atitudes transmitidas às crianças e jovens através do tom de voz e da linguagem corporal das figuras parentais (Darling \& Steinberg, 1993). De acordo com Baumrind (1966), existem três tipos de estilos parentais: permissivo, autoritário e democrático. 0 estilo permissivo é atribuído quando os pais se comportam de uma forma não punitiva, aceitando os desejos e ações da criança. As figuras parentais têm poucas exigências tanto a nível do controlo do comportamento como na responsabilidade de tarefas, não incentivando a seguir regras socialmente estabelecidas. No estilo parental autoritário, o controlo e a avaliação dos comportamentos da criança são feitos de acordo com um padrão estabelecido geralmente por uma figura de autoridade parental e existem fracas trocas afetivas. Neste caso, a criança deve aceitar as ordens, restringindo a sua autonomia. Por fim, os pais que adotam um estilo parental democrático reconhecem os interesses da criança e a autonomia da mesma e têm regras e disciplina estabelecendo padrões de condutas adaptativas. São figuras parentais que usam a razão e o poder, bem como o reforço, o afeto e a modelagem para conseguirem comportamentos ajustados.

Os padrões de comportamento que possibilitam a adaptação ao contexto onde a criança se insere desenvolvem-se desde cedo. Estes padrões de comportamentos são transmitidos às crianças através das suas interações sociais e interpessoais, nomeadamente com a família e no contexto escolar (Saud \& Tonelotto, 2005). Tendo em conta a perspetiva sistémica da família, a presença de bons modelos que fomentem a autonomia, a regulação emocional, a comunicação e o afeto, resulta na promoção de comportamentos pró-sociais dos jovens. Pelo contrário, a negligência, a falta de comunicação, famílias disfuncionais e abuso de substâncias por parte dos pais, são considerados fatores de risco, podendo causar condutas desviantes nos jovens (Tomé et al., 2015). Neste sentido, os estilos parentais contribuem para o desenvolvimento socio-emocional dos jovens e constituem-se como fatores importantes na compreensão dos seus comportamentos (Baumrind, 1967; Weber, 2017).

A partir da adolescência, os jovens tendem a ter uma maior aproximação aos pares, no entanto, a presença dos pais continua a desempenhar uma fonte de apoio importante para os jovens (Hoeve et al., 2011). Para Sangawi et al. (2016), a confiança e a segurança transmitidas pelas figuras parentais facilitam o estabelecimento de relações interpessoais dos jovens com os pares. As habilidades sociais que os jovens desenvolvem também são, em parte, delineados pelos estilos parentais que os pais adotam, havendo uma possibilidade de desenvolver habilidades sociais adaptativas na presença de um estilo parental democrático (Maia \& Soares, 2019). O laço existente entre o jovem e os seus cuidadores é capaz de potenciar um comportamento socialmente desejável e, consequentemente, um menor envolvimento em comportamentos de risco (Formiga et al., 2013).

A família é o contexto onde são feitas as principais aprendizagens tendo assim um papel importante no desenvolvimento de comportamentos socialmente ajustados, por exemplo, o consumo de álcool nos jovens estudantes continua a ser um problema social e com consequências psicológicas, sociais e físicas para a saúde. 0 ambiente familiar é considerado um fator protetor quando existe um bom suporte familiar, uma monitorização parental adequada e uma boa comunicação entre os elementos (Baumrind, 1991). Assim, o estilo parental democrático é considerado um fator fundamental na minimização do consumo de álcool dos jovens (Cerutti, Ramos, \& Arfimon, 2015). Em contrapartida, o contexto familiar pode também potenciar o maior consumo de álcool nos mesmos, como é o caso da presença de estilos parentais autoritários (Riquelme et al., 2018) e permissivos (Charfi et al., 2018; Hoffman \& Bahr, 2014).

Outros comportamentos desadaptativos podem acontecer em jovens que tenham vivido com figuras parentais que adotaram estilos parentais menos adequados dificultando a sua adaptação a novos contextos. Gallo e Williams (2008) verificaram que existem diversas caraterísticas que se associam ao comportamento desviante, nomeadamente dificuldades de socialização, impulsividade, envolvimento com pares desviantes e uso precoce de tabaco e bebidas alcoólicas.

Alguns estudos apontam para a presença de um estilo parental democrático que parece funcionar como fator de proteção no menor envolvimento em consumos de álcool, tabaco e outras drogas dos jovens, uma vez que os pais tendem a transmitir comportamentos pró-sociais através da forma positiva com que lidam com os filhos, além de contribuírem para um desenvolvimento adaptativo dos mesmos (Church et al., 2012; Eiden et al., 2016; Tondowski et al., 2015). Há um efeito contrário na adoção de estilos parentais negativos (Benchaya et al., 2011), nomeadamente o estilo parental autoritário (Gershoff \& Grogan-Kaylor, 2016) e o estilo parental permissivo (Hoeve et al., 2011; Sahed, 2016), uma vez que os jovens apresentam mais consumos adictivos. Assim sendo, verifica-se que o estilo parental adotado pelos pais pode ter um efeito tanto no consumo de álcool como no desenvolvimento de comportamento desviante, nomeadamente no comportamento adictivo.

0 consumo de álcool nos jovens adultos leva ao desenvolvimento de outro tipo de comportamentos de 
risco (Pérez-Gomez, Lanziano, Reyes-Rodríguez, MejíaTrujillo, \& Cardozo-Macías, 2018), como o consumo de tabaco e de drogas (Fergusson et al., 1994). No estudo de Kirby e Barry (2012), o comportamento adictivo dos jovens foi predito pelo consumo de álcool, tendo sido encontrado o mesmo resultado em outros estudos (Nichter et al., 2010; Obradovic, 2017; Roche et al., 2019). O consumo de álcool parece estar na origem de outros comportamentos desviantes (Xue et al., 2009), nomeadamente comportamentos delinquentes, agressivos (Díaz-García, \& Moral-Jiménez, 2018), bem como comportamentos de vandalismo e roubos, entre outros (Fonseca, 2013; Skara et al., 2008). Deste modo, o padrão de comportamentos aditivos e autodestrutivos podem ser englobadas ações que correspondem a violações das normas legais, como a venda de droga, e das normas sociais, como o consumo de tabaco, bebidas alcoólicas e outras drogas (Sanches \& Gouveia-Pereira, 2010).

Assim sendo, o objetivo deste estudo foca-se na análise do efeito mediador do consumo de álcool na relação existente entre os estilos parentais e o desenvolvimento de comportamento desviante.

\section{Objetivos}

A presente investigação tem como objetivo geral analisar o papel dos estilos parentais e do consumo de álcool no comportamento desviante em jovens adultos. Especificamente, pretende-se analisar as associações existentes entre as variáveis em estudo, nomeadamente os estilos parentais, o consumo de álcool e o comportamento desviante. Além disso, tenciona-se analisar o efeito mediador do consumo de álcool na ligação entre os estilos parentais e o comportamento aditivo e autodestrutivo.

\section{Método}

\section{Participantes}

No estudo participaram 1044 jovens de uma universidade do norte de Portugal que frequentavam o $1^{\circ}$ ou $2^{\circ}$ ciclo de estudos, dos quais $932(89.3 \%)$ o $1^{\circ}$ ciclo, $85(8.1 \%)$ o $4^{\circ}$ ano de licenciatura ou o $1^{\circ}$ ano de mestrado e 21 (2\%) o $2^{\circ}$ ano de mestrado. Dos participantes, 277 (26.5\%) são do sexo masculino e 767 (73.5\%) do sexo feminino, com idades compreendias entre os 18 e 25 anos $(M=19.78$; $D P=1.68)$.

\section{Instrumentos}

\section{Questionário Sociodemográfico}

Este questionário tem o objetivo de recolher dados acerca dos participantes, nomeadamente o sexo, a idade, a escolaridade do pai e da mãe, entre outros elementos.

\section{Parenting Styles \& Dimensions Questionnaire - Short Version (PSDQ)}

O PSDQ (Robinson et al., 1995, adaptado por Nunes e Mota, 2018) permite avaliar a perceção dos jovens quanto aos estilos parentais. É uma escala de autorrelato que contêm 32 itens apresentados numa escala tipo Likert que varia de 1 (Nunca) a 5 (Sempre) e duas versões (pai e mãe). Está organizado em três dimensões globais, nomea- damente o estilo democrático, o autoritário e o permissivo, sendo que cada uma possui diferentes subescalas. Assim sendo, o estilo democrático possui três subescalas, o "apoio e afeto" (itens 1, 7, 12, 14 e 27), a "regulação" (5, 11, 25, 29 e 31) e a "cedência de autonomia" (3, 9, 18, 21 e 22). O estilo autoritário possui a "coerção física" $(2,6,19$ e 32), a "hostilidade verbal" (13, 16, 23 e 30) e a "punição" (4, 10, 26 e 28). Por fim, o estilo permissivo é constituído por uma subescala, a "indulgência" (8, 15, 17, 20 e 24). De acordo com a adaptação do instrumento segundo Nunes e Mota (2018), o item 23 ajustou melhor na dimensão regulação do que na dimensão hostilidade pelo que se procedeu a esta alteração na presente investigação pela presença de melhoria em termos de índices de ajustamento. Relativamente às análises psicométricas verificou-se um valor de alfa de Cronbach de .89 para a figura paterna e .84 para a figura materna. Relativamente às dimensões para o pai e mãe, respetivamente: o afeto e apoio $.90 / .86$, a regulação $.87 / .83$, a cedência de autonomia $.88 / .83$, a coerção física $.66 / .71$, a hostilidade verbal $.56 / .57$, a punição $.58 / .57$ e, por fim, a dimensão indulgência $.57 / .56$. Relativamente às analises fatoriais confirmatórias os valores foram os seguintes para o pai $\left(\chi i^{2}(443)=1833.255 ; p=.000 ; \chi i^{2} / g l=4.138\right.$; $\mathrm{CFI}=.909 ; \mathrm{NFI}=.883 ; \mathrm{SRMR}=.078 ; \mathrm{RMSEA}=.055)$ e para a mãe $\left(\chi i^{2}(443)=1674.703 ; p=.000 ; \chi i^{2} / g l=3.780 ; \quad C F I=.902 ;\right.$ $\mathrm{NFI}=.872 ; \mathrm{SRMR}=.071 ; \mathrm{RMSEA}=.052$ ).

\section{Escala de Comportamentos Desviantes (ECD)}

A ECD (Gouveia-Pereira \& Carita, 2005) permite avaliar a frequência dos comportamentos desviantes. É constituído por 41 itens de autorresposta através de uma escala de Likert de seis pontos sendo 1 (muitas vezes) e 6 (nunca) divididos por seis dimensões: comportamentos adictivos e autodestrutivos (item 18, 29, 6, 25, 26, 30, 28, 19 e 5); roubos (item 11, 12, 13, 38, 39, 40, 23 e 14); comportamentos violentos (item 20, 21, 9, 22 e 27); comportamentos agressivos dirigidos a professores (item 36, 35, 37 e 34); comportamentos disruptivos na escola (item 1, 3, 2, 10 e 8); e mentiras acerca da idade (item 4 e 41). Foi realizada a inversão de todos os itens de forma a obter valores por ordem crescente de presença. Após realizar todas as análises psicométricas verificou-se que apenas a dimensão "comportamento adictivo e autodestrutivo" (onde se destacam consumos de substâncias licitas e ilícitas, e comportamentos de risco no consumo e venda) apresentava um alfa de Cronbach aceitável de .71 (roubos=.42; comportamentos violentos=.31; comportamentos agressivos dirigidos a professores=.05; comportamento disruptivo na escola $=.54$; mentiras acerca da idade $=.54$ ) e um índice de ajustamento aceitável $\left(\chi i^{2}(23)=217.667 ; p=.000 ; \chi i^{2} / g l=9.464 ; C F I=.910\right.$; $\mathrm{NFI}=.901$; SRMR=.052; RMSEA=.090) pelo que se procedeu à retirada das restantes dimensões na presente investigação.

\section{Alcohol Use Disorders Identification Test - (AUDIT)}

O AUDIT (Saunders et al., 1993, adaptado por Cunha, 2002) permite avaliar os tipos de consumo de álcool. É constituído por 10 itens, cujas respostas são dadas numa escala tipo Likert de 0 a 4, sendo que, apesar da sua divisão ser feita em três dimensões (padrão de consumo, dependência de consumo e consequências de consumo), optou-se por 
utilizar o somatório da escala, passando a ser usada como variável continua. A escala geral apresentou uma boa consistência interna, sendo o alfa de Cronbach .91 e o índice de ajustamento $\left(\chi i^{2}(33)=113,046 ; p=.000 ; \chi i^{2} / g l=3.426\right.$; $\mathrm{CFI}=.987 ; \mathrm{NFI}=.981 ; \mathrm{SRMR}=.0229 ; \mathrm{RMSEA}=.048$ ).

\section{Procedimento}

Realizou-se a elaboração do protocolo, salientando de que todos os instrumentos se encontram validados para a população portuguesa. Solicitou-se a aprovação da Comissão de Ética da instituição de ensino superior onde foi realizado o estudo. Após todas as autorizações terem sido aceites, procedeu-se ao contacto direto com os professores de forma a agendar as recolhas dos dados. A recolha dos dados da presente investigação foi realizada numa instituição de ensino superior do norte de Portugal de forma aleatória (mediante autorização das escolas, aceitação das direções e voluntariedade dos alunos), tendo sido solicitado a cada participante a assinatura do Consentimento Livre e Esclarecido e dado a conhecer o objetivo do estudo. A recolha decorreu num único momento (tempo médio de preenchimento de 30 minutos), evidenciando o cariz transversal do estudo e decorreu com a presença do investigador. 0 presente estudo teve em conta os princípios éticos e deontológicos da psicologia.

\section{Análise de dados}

Os dados foram tratados a partir do programa estatístico Statistical Package for Social Sciences (SPSS). Inicialmente, procedeu-se à limpeza da base de dados (removeramse, no total, 156 participantes) de forma a salvaguardar a qualidade dos dados, identificando e excluindo os missings e os outliers através da análise da distância de Mahalanobis. Posteriormente, a distribuição normal dos dados foi verificada através das medidas de assimetria (skewness) e achatamento (kurtose), do teste Kolmogorov-Smirnov e dos histogramas. Após ser verificada a normalidade procedeu-se à análise dos dados utilizando testes paramétricos. Procedeu-se à análise da consistência interna, através dos valores de alpha de Cronbach e realizaram-se análises confirmatórias de $1^{a}$ ordem através do programa estatístico AMOS (versão 23.0). De seguida, realizaram-se análises descritivas que envolveu o cálculo de frequências, médias e desvios padrões. Seguidamente, foram executadas as análises correlacionais ( $r$ de Pearson), de forma a verificar a associação existente entre as diferentes variáveis em estudo. Por fim, verificou-se o papel mediador do consumo de álcool na ligação entre os estilos parentais e o comportamento desviante através do programa AMOS.

\section{Resultados}

Associação entre estilos parentais, consumo de álcool e comportamento desviante em estudantes do ensino superior

De forma a analisar as associações entre as variáveis, estilos parentais, consumo de álcool e comportamento desviante em jovens adultos do ensino superior procedeu-se à realização de análises correlacionais. Os resultados das análises inter-escalas, as médias e os respetivos desvios padrões encontram-se reportados na Tabela 1. Relativamente à associação entre a dimensão da escala do comportamento desviante, comportamento adictivo e autodestrutivo, e dos estilos parentais paternos, os resultados apontam a existência de correlações significativas, negativas de baixa magnitude, nas dimensões "afeto e apoio" $(r=-.12, p<.01)$ e "cedência de autonomia" $(r=-.10, p<.01)$ e correlações positivas de magnitude baixa nas dimensões "coerção física" $(r=.07, p<.05)$, "hostilidade verbal" $(r=.12, p<.01)$, “punição" $(r=.13, p<.01)$ e "indulgência” $(r=.12, p<.01)$. No que diz respeito à associação entre a dimensão "comportamento adictivo e autodestrutivo" com os "estilos parentais maternos", os resultados apontam para correções significativas negativas e de baixa magnitude nas dimensões "afeto e apoio" ( $r=-.15, p<.01)$, "regulação" $(r=-.08$, $p<.05)$ e "cedência de autonomia" $(r=-.12, p<.01)$. Por outro lado, existem correlações significativas positivas e de baixa magnitude nas dimensões "coerção física” $(r=.09, p<.01)$, hostilidade verbal” ( $r=.10, p<.01)$, “punição" $(r=.12, p<.01)$ e "indulgência" $(r=.14, p<.01)$. Além disso, a associação entre a dimensão "comportamento adictivo e autodestrutivo" e o "consumo de álcool" demonstra a existência de uma correlação significativa positiva de elevada magnitude $(r=.65, p<.01)$.

Relativamente à associação entre os "estilos parentais paternos" e o "consumo de álcool”, verifica-se uma correlação significativa negativa entre o "consumo de álcool" e a dimensão "afeto e apoio" (estilo parental democrático) $(r=-.06, p<.05)$. Em contrapartida, o consumo de álcool apresenta correlações significativas negativas de baixa magnitude com as dimensões do estilo parental autoritário, nomeadamente a "coerção física" $(r=.07, p<.05)$, "hostilidade verbal” $(r=.13, p<.01)$, punição $(r=.11, p<.01)$ e com a dimensão do estilo parental permissivo, "indulgência" $(r=.11, p<.01)$. No que respeita à associação entre os "estilos parentais maternos" e o "consumo de álcool" os resultados indicam a existência de uma correlação negativa e significativa de baixa magnitude entre o consumo de álcool e a dimensão do estilo parental democrático "afeto e apoio" $(r=-.09, p<.01)$ e correlações negativas significativas de baixa magnitude com as dimensões do estilo parental autoritário coerção física" ( $r=.10, p<.01)$, "hostilidade verbal”, $(r=.13, p<.01)$ "punição" $(r=.11, p<.01)$ e do estilo parental permissivo "indulgência” $(r=.14, p<0.1)$.

\section{Efeito mediador do consumo de álcool na associa- ção entre os estilos parentais e o comportamento desviante em jovens adultos}

De modo a analisar o efeito preditivo dos estilos parentais positivos (estilo democrático - dimensão afeto e apoio, regulação e cedência de autonomia) e estilos parentais negativos (autoritário - coerção física, hostilidade verbal e punição/estilo permissivo - indulgência) no desenvolvimento de comportamento desviante, bem como testar o efeito mediador do consumo de álcool desempenhado nessa associação, procedeu-se, através do programa AMOS, à realização de equações estruturais. Importa referir que foi testado o papel mediador do consumo de álcool, tendo em conta os estilos parentais maternos e paternos separadamente. 


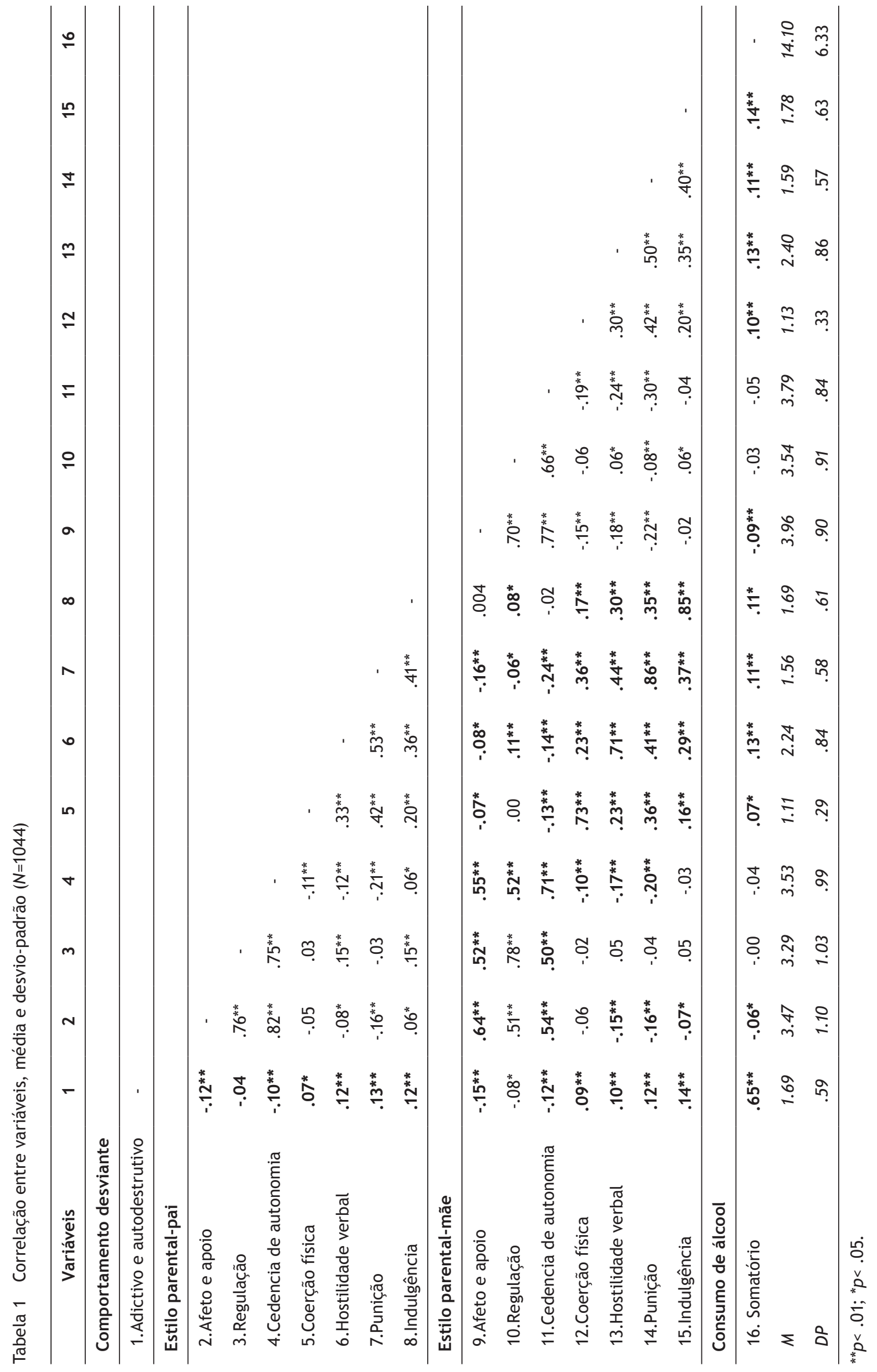


Em um primeiro momento, analisou-se a associação entre as variáveis no modelo referente à figura materna, tendo obtido os seguintes resultados significativos $(p<.05)$ : o estilo parental materno positivo (democrático) prediz negativamente o comportamento desviante $(\beta=-.15)$ e o estilo parental materno negativo (autoritário e permissivo) prediz positivamente o comportamento desviante $(\beta=.17)$. Por sua vez, o estilo parental materno positivo prediz o consumo de álcool de forma negativa $(\beta=-.08)$ e o estilo parental materno negativo prediz o consumo de álcool de forma positiva $(\beta=.17)$. 0 consumo de álcool prediz de forma positiva o comportamento desviante $(\beta=.64)$. Posteriormente, introduziu-se a variável mediadora (consumo de álcool) no modelo final e, com recurso ao procedimento bootstrapping, verificou-se que a dimensão referente ao estilo parental materno negativo prediz de forma positiva e significativa o consumo de álcool $(\beta=.17)$ que, por sua vez, prediz o comportamento desviante $(\beta=.64)$. Assim, verificou-se a existência de uma mediação total positiva do consumo de álcool na associação entre o estilo parental materno negativo e o comportamento desviante ( $\mathrm{SE}=.025 ; \beta=.107 ; p=.001$; IC $90 \%[.065, .148])$, uma vez que a ligação entre o estilo parental materno negativo e o comportamento desviante (Binicial=.17) deixou de ser significativa. 0 modelo apresenta valores de índice de ajustamento adequados: $\left(\chi^{i^{2}}(24)=\right.$ $138.604, p=.000, \chi i^{2} / \mathrm{gl}=5.775 ; \mathrm{CFI}=.965, \mathrm{NFI}=.959$, SRMR $=.049$, RMSEA $=.068$ ) (Figura 1).

No que concerne a figura paterna, verificou-se que o estilo parental paterno positivo prediz negativamente o comportamento desviante $(\beta=-.11)$ e que o estilo parental paterno negativo prediz de forma positiva o comportamento desviante ( $\beta=.17)$. Também se observa que o estilo parental paterno negativo prediz de forma positiva o consumo de álcool $(\beta=.16)$; no entanto, a ligação entre o estilo parental paterno positivo e o consumo de álcool não apresenta significância ( $\beta=-.05 ; p=.157)$. À semelhança do modelo relativo à figura materna, o consumo de álcool prediz de forma positiva o comportamento desviante $(\beta=.65)$. Após a introdução da variável mediadora "consumo de álcool", verificou-se que a ligação entre o estilo parental paterno negativo e o comportamento desviante se manteve significativa, no entanto, perdeu magnitude ( $\beta$ inicial=.17; $\beta$ final=.06) observando-se uma mediação parcial positiva ( $\mathrm{SE}=.024 ; \beta=.101$; $p=.001 ;$ IC $90 \%$ [.062, .142]). Neste sentido, o consumo de álcool partilha o efeito da relação entre o estilo parental negativo paterno e o comportamento adictivo e autodestrutivo, explicando parte do relacionamento entre as variáveis. 0 modelo apresenta valores de ajustamento adequados $\left(\mathrm{xi2}(23)=145.540, p=.000, \chi i^{2} / \mathrm{gl}=6.328 ; \mathrm{CFI}=.968\right.$, $\mathrm{NFI}=.962$, SRMR=.053, RMSEA=.071) (Figura 2).

\section{Discussão}

A presente investigação assumiu como objetivo principal testar o papel dos estilos parentais no comportamento desviante em jovens adultos que frequentassem o ensino superior, averiguando o efeito mediador do consumo de álcool na associação anterior.

0 resultado obtido vai ao encontro do que era esperado, uma vez que os jovens que vivenciam um estilo parental autoritário caraterizados por uma baixa autoestima, insegurança, baixas habilidades sociais e maiores

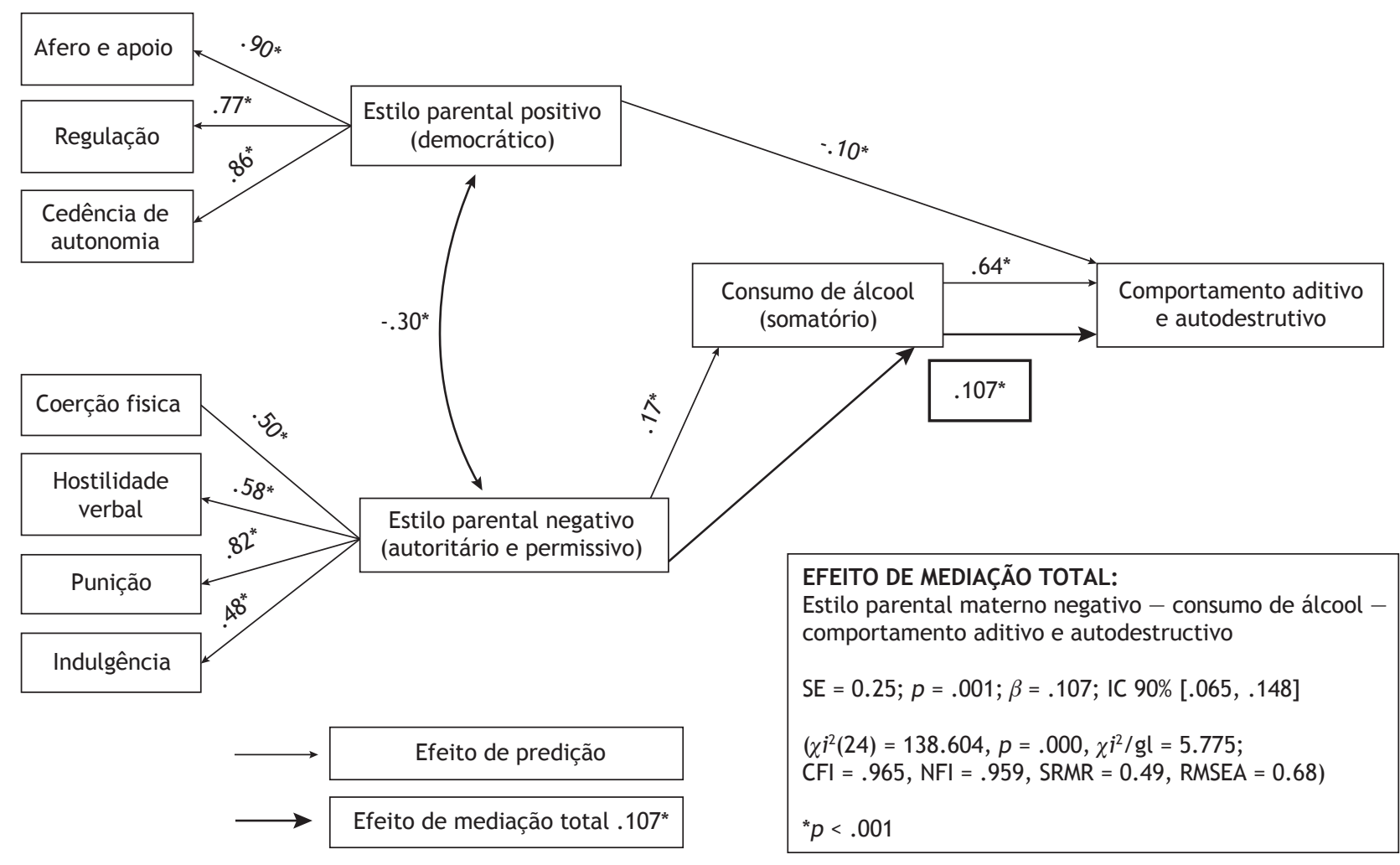

Figura 1. Modelo representativo do efeito mediador do consumo de álcool na associação entre os estilos parentais maternos e o comportamento adictivo e autodestrutivo 


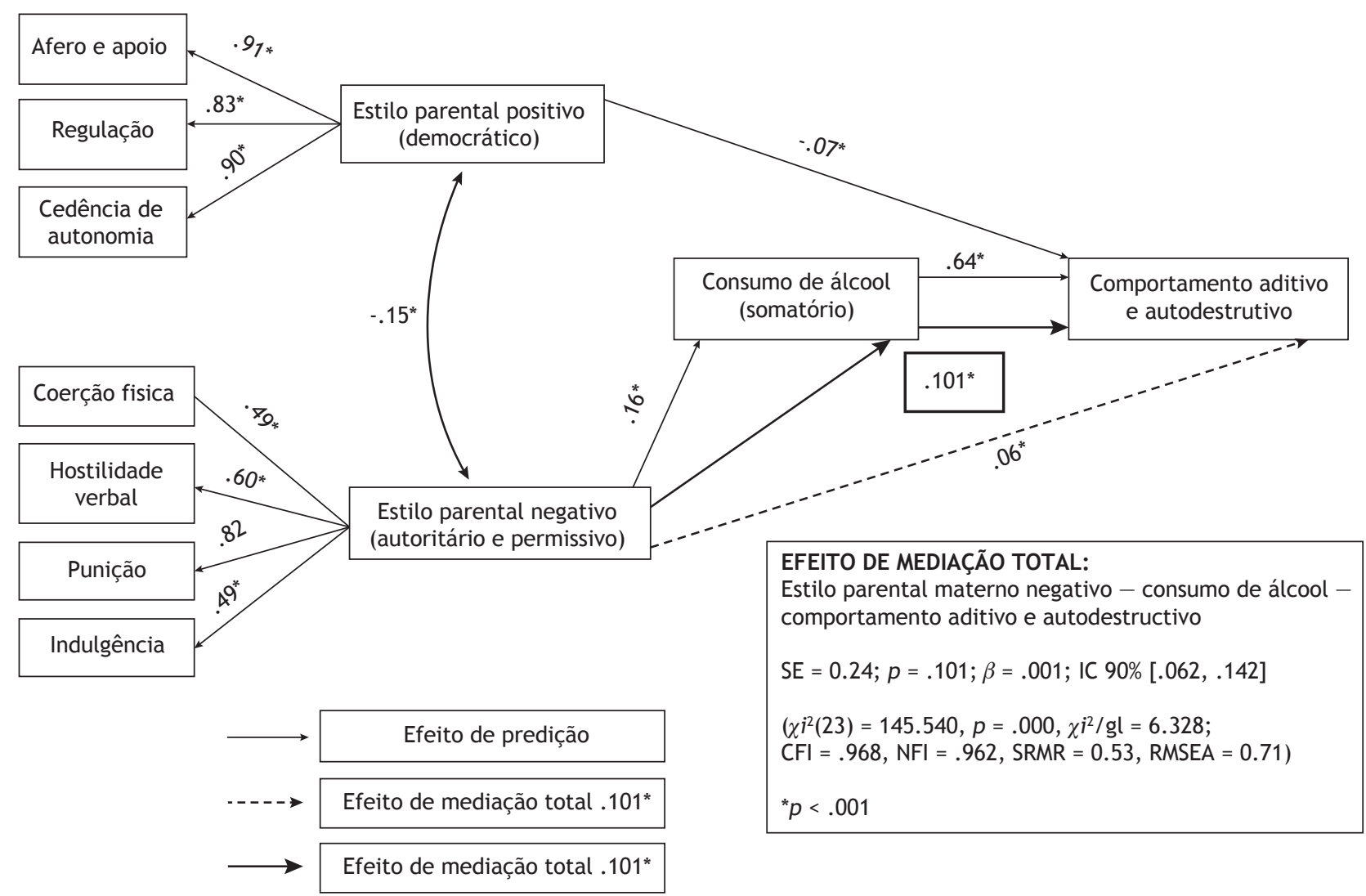

Figura 2. Modelo representativo do efeito mediador do consumo de álcool na associação entre os estilos parentais paternos e o comportamento adictivo e autodestrutivo

níveis de ansiedade demonstram uma maior predisposição no envolvimento em comportamentos adictivos (Hoeve et al., 2011). O mesmo se verifica nos jovens cujos pais apresentam um estilo parental permissivo, demonstrando que os jovens cujos pais têm um estilo parental negativo envolvem-se em mais comportamentos de risco como forma de inclusão e procura de emoções positivas. No que concerne o estilo parental democrático, verifica-se que o mesmo prediz de forma negativa o comportamento adictivo e autodestrutivo nos jovens. Este resultado era expectável, uma vez que a existência de afeto e responsividade parental parecem ter um impacto positivo no desenvolvimento dos mesmos, além de que, nestes casos, os jovens têm menos necessidade em procurar emoções positivas, menos receio da rejeição e menor tendência em associar-se a pares desviantes. Vários estudos concluíram os mesmos resultados, em que o estilo parental democrático funciona como fator protetor (Church et al., 2012; Eiden et al., 2016; Tondowski et al., 2015) e os estilos parentais negativos (autoritário e permissivo) como fatores de risco (Cutrín et al., 2017; Gershoff \& Grogan-Kaylor, 2016; Mowen \& Schroeder, 2015; Sahed, 2016) no que concerne ao envolvimento em comportamentos adictivos e autodestrutivos dos jovens.

Ainda face aos resultados observados, verificou-se que os estilos parentais negativos maternos e paternos predizem positivamente o consumo de álcool nos jovens. Por sua vez, o estilo parental democrático materno prediz de forma negativa o consumo de álcool. Este resultado era esperado, uma vez que a presença de um ambiente familiar positi- vo, pautado por cuidado, afeto, segurança e responsividade associa-se a um menor envolvimento dos jovens com 0 álcool. Este resultado demonstra que a figura materna apresenta uma postura mais próxima e eficaz na transmissão de comportamentos pró-sociais dos filhos, nomeadamente no menor envolvimento com álcool, além de poder ser considerada como a figura com maior envolvimento parental, maior acompanhamento e eficácia na supervisão dos mesmos (Maia \& Soares, 2019). Este contexto familiar facilita o desenvolvimento de estratégias de coping adequadas, de melhores habilidades sociais e numa maior tendência para se agrupar com pares pró-sociais. Vários estudos concluíram o mesmo resultado, em que a presença de um estilo parental autoritário e permissivo (Charfi et al., 2018; Hoffman \& Bahr, 2014; Riquelme et al., 2018) potencia o maior risco de envolvimento com álcool, ao contrário da presença de um estilo parental democrático (Maia \& Soares, 2019; Sangawi et al., 2016).

Mais especificamente, e tendo em conta o modelo da figura materna, verifica-se que o comportamento adictivo e autodestrutivo é predito negativamente pelo estilo parental materno positivo (democrático). Por outro lado, observa-se que o comportamento adictivo e autodestrutivo é predito positivamente pelos estilos parentais maternos negativos (autoritário e permissivo). O mesmo resultado foi encontrado no estudo de Benchaya et al. (2011), no qual o estilo parental democrático se associa de forma negativa com o desenvolvimento de comportamento adictivo, ocorrendo o oposto com os estilos parentais negativos. No seguimento 
da análise que diz respeito à figura materna, constata-se a existência de uma mediação total do consumo de álcool na associação entre o estilo parental materno negativo e o comportamento adictivo e autodestrutivo. Deste modo, observou-se que o estilo parental negativo (autoritário e permissivo) prediz positivamente um maior consumo de álcool e que este prediz positivamente o envolvimento em comportamentos adictivos e autodestrutivos. Neste sentido, no presente estudo o consumo de álcool é considerado um fator de risco e um condutor para o envolvimento com outros comportamentos adictivos e autodestrutivos nos jovens adultos.

No que concerne a figura paterna, os resultados evidenciam que um estilo parental paterno positivo (democrático) prediz negativamente o comportamento adictivo e autodestrutivo. Por sua vez, a presença de um estilo parental negativo (autoritário e permissivo) prediz positivamente o comportamento adictivo e autodestrutivo. Assim, verifica-se que jovens cujas figuras paternas apresentem um estilo parental negativo poderão ter mais facilidade em envolver-se em comportamentos adictivos e autodestrutivos. Por fim, no modelo referente à figura paterna, observa-se a existência de uma mediação parcial do consumo de álcool na associação entre o estilo parental paterno negativo e o desenvolvimento de comportamento adictivo e autodestrutivo. Assim, jovens que tenham uma figura paterna com um estilo parental negativo poderão ser mais suscetíveis ao envolvimento com o álcool e com comportamento adictivos e autodestrutivos. Por sua vez, o maior envolvimento com o consumo de álcool, com mais risco (um consumo mais frequente e em maiores quantidades) poderá levar a um maior envolvimento com outros comportamentos adictivos e autodestrutivos nos jovens (e.g., Díaz-García, \& Moral-Jiménez, 2018; Pérez-Gomez et al., 2018). Roche et al. (2019) defendem que a presença de um consumo de álcool de maior risco pode levar ao desenvolvimento de outros comportamentos de risco, como o envolvimento com o tabaco e drogas ilícitas.

No presente estudo, jovens que se desenvolvem numa família cujas figuras paternas denotem estilos parentais negativos parecem estar mais predispostos a envolverem-se em consumos de risco de álcool. Este resultado pode ser explicado pelo facto de que figuras parentais que valorizam a autoridade ou a permissividade promovem nos jovens sentimentos de insegurança, baixa autoestima e fracas habilidades sociais que poderão revelar uma maior necessidade em consumir bebidas alcoólicas proporcionando uma sensação de inclusão e uma forma de lidar com os problemas. Por sua vez, o consumo de álcool poderá potenciar um maior envolvimento com outros comportamentos adictivos, como o consumo de drogas. Neste sentido, os resultados parecem refletir a importância de um ambiente familiar positivo, pautado por um estilo parental democrático face ao menor envolvimento com o álcool e com outros comportamentos adictivos e autodestrutivos dos jovens.

\section{Implicações práticas, limitações e pistas futuras}

O estudo assume um contributo significativo, na medida em que reforçou a importância do estilo parental democrático no que diz respeito ao menor envolvimento dos jovens em comportamentos de risco. Por sua vez, permitiu destacar o papel do consumo de álcool na associação entres os estilos parentais e o comportamento adictivo e autodestru- tivo. Permitiu um maior conhecimento na área da parentalidade e do consumo de substâncias dos jovens o que poderá ajudar no desenvolvimento de campanhas preventivas que poderão incidir na aquisição de competências sociais e de estratégias de coping adaptativas nos estudantes universitários e também na importância da sensibilização destas famílias no que diz respeito à importância das suas práticas parentais no acompanhamento dos jovens, com vista ao desenvolvimento adaptativo.

Quanto às limitações do estudo, verifica-se a presença de uma amostra apenas universitária, embora fosse de interesse particular no presente estudo, onde se verificam inúmeros processos associados ao comportamento desviante, todavia a mesma é maioritariamente feminina, sendo a recolha aleatória que carateriza genericamente este contexto. O uso de instrumentos de autorrelato constitui, desde logo, uma limitação face à desejabilidade social, particularmente quando as temáticas convergem para o comportamento desviante. Acresce às limitações o cariz transversal da investigação, não permitindo a inferência de relações de causalidade e, ainda, as fracas qualidades psicométricas dos instrumentos PSDQ e ECD. Em termos de pistas futuras, seria pertinente abordar outras variáveis, replicar o estudo em outras áreas geográficas do país e envolvendo adolescentes, usar um instrumento mais ajustado no que respeita a avaliação dos comportamentos desviantes e realizar um emparelhamento entre as perceções dos jovens e as perspetivas das figuras parentais em termos de estilos parentais.

\section{Reconhecimento}

Esta investigação é parcialmente suportada pelo Centro de Psicologia da Universidade do Porto, Fundação para a Ciência e Tecnologia (FCT UIDB/00050/2020).

\section{Referências}

Baumrind, D. (1966). Effects of authoritative parental control on child behavior. Child Development, 37(4), 887-907. https://doi. org/10.2307/1126611

Baumrind, D. (1967). Childcare practices anteceding three patterns of preschool behavior. Genetic Psychology Monographs, 75(1), 43-88.

Baumrind, D. (1991). The influence of parenting styles on adolescent competence and substance use. Journal of Early Adolescence, 11(1), 56-95. https://doi.org/10.1177/0272431691111004

Benchaya, M. C., Bisch, N. K., Moreira, T. C., Ferigolo, M., \& Barros, H. M. T. (2011). Non-authoritative parents and impact on drug use: The perception of adolescent children. Jornal de Pediatria, 87(3), 238-244. https://doi.org/10.2223/JPED.2089

Cerutti, F., Ramos, S. P., \& Arfimon, I. I. L. (2015). A implicação das atitudes parentais no uso de drogas na adolescência. Acta Colombiana de Psicología, 18(2), 73-181. https://doi.org/10.14718/ ACP.2015.18.2.15

Charfi, N., Turki, M., Smaoui, N., Bouali, M. M., Omri, S., Zouari, L., Thabet, J. B., \& Maâlej, M. (2018). Alcohol use and associated environmental factors among middle and high school students in Sfax (Tunisia). International Journal of Mental Health and Addiction, 1-16. https://doi.org/10.1007/s11469-018-9969-6

Church, W. T., Tomek, S., Bolland, K. A., \& Hooper, L. M. (2012). A longitudinal examination of predictors of delinquency: An analysis of data from the mobile youth survey. Children and Youth Services Review, 34(12), 2400-2408. https://doi.org/10.1016/j. childyouth.2012.09.007 
Cunha, J. (2002). Validação da versão portuguesa dos Questionários AUDIT e Five-Shot para identificação de consumo excessivo de álcool. Internato Complementar de Clínica Geral da Zona Sul.

Cutrín, O., Fraguela, J. A. G., \& Sobral, J. (2017). Gender differences in the influence of parenting on youth antisocial behavior through deviant peers. The Spanish Journal of Psychology, 20(58), 1-10. https://doi.org/10.1017/sjp.2017.53

Darling, N., \& Steinberg, L. (1993). Parenting style as context: An integrative model. Psychological Bulletin, 113(3), 487-496. https://doi.org/10.1037/0033-2909.113.3.487

Díaz García N., \& Moral Jiménez M. de la V. (2018). Consumo de alcohol conducta antisocial e impulsividad en adolescentes españoles. Acta Colombiana de Psicología, 21(2), 110-120. https:// doi.org/10.14718/ACP.2018.21.2.6

Eiden, R. D., Lessard, J., Colder, C. R., Livingston, J., Casey, M., \& Leonard, K. E. (2016). Developmental cascade model for adolescent substance use from infancy to late adolescence. Developmental Psychology, 52(10), 1619-1633. https://doi.org/10.1037/ dev0000199

Fergusson, D. M., Lynskey, M. T., \& Horwood, L. J. (1994). Alcohol consumption and associated problems in a birth cohort of 15year olds. New Zealand Medical Journal, 107(977), 167-170.

Fonseca, A. C. (2013). Consumo de drogas e comportamentos antissociais na adolescência: Que relação? Revista Portuguesa de Pedagogia, 47(1), 157-176. https://doi.org/10.14195/16478614_47-1_8

Formiga, N. S., Melo, G., \& Leme, J. (2013). Pares sócio-normativos, orientação cultural, hábitos de lazer e condutas desviantes: Verificação de um modelo teórico em jovens. Revista de Psicologia Universidad de Antioquia, 5(1), 7-26.

Gallo, A. E., \& Williams, L. C. A. (2008). A escola como fator de proteção à conduta infracional de adolescentes. Cadernos de Pesquisa, 38(133), 41-59.

Gershoff, E. T., \& Grogan-Kaylor, A. (2016). Spanking and child out-comes: Old controversies and new meta-analyses. Journal of Family Psychology, 30(4), 453-469. https://doi.org/10.1037/ fam0000191

Gouveia-Pereira, M., \& Carita, A. (2005). Percepções de justiça em contexto escolar e familiar e sua relação com o exercício da cidadania e com comportamentos desviantes (Manuscrito não publicado). Instituto Superior de Psicologia Aplicada, Lisboa.

Hoeve, M., Dubas, J. S., Gerris, J. R. M., Laan, P. H., \& Smeenk, W. (2011). Maternal and paternal parenting styles: Unique and combined links to adolescent and early adult delinquency. Journal of Adolescence, 34(5), 813-827. https://doi.org/10.1016/j. adolescence.2011.02.004

Hoffman, J. P., \& Bahr, S. J. (2014). Parenting style, religiosity, peer alcohol use, and adolescent heavy drinking. Journal of Studies on Alcohol and Drugs, 75(2), 222-227. https://doi.org/10.15288/ jsad.2014.75.222

Kirby, T., \& Barry, A. E. (2012). Alcohol as a gateway drug: A study of us $12^{\text {th }}$ graders. Journal of School Health, 82(8), 371-379. https://doi.org/10.1111/j.1746-1561.2012.00712.x

Maia, F. A., \& Soares, A. B. (2019). Diferenças nas práticas parentais de pais e mães e a percepção dos filhos adolescentes. Estudos Interdisciplinares em Psicologia, 10(1), 59-82. https://doi. org/10.5433/2236-6407.2019v10n1p59

Mowen, T. J., \& Schroeder, R. D. (2015). Maternal parenting style and delinquency by race and the moderating effect of structural disadvantage. Sage Journal, 50(2), 139-159. https://doi. org $/ 10.1177 / 0044118 \times 15598028$

Nichter, M., Nichter, M., Carkoglu, A., \& Lloyd-Richardson, E. (2010). Smoking and drinking among college students: "It's a package deal". Drug and Alcohol Dependence, 106(1), 16-20. https://doi. org/10.1016/j.drugalcdep.2009.07.025

Nunes, F., \& Mota, C. P. (2018). Parenting styles and dimensions questionnaire - adaptação da versão portuguesa de heterorrelato. Revista Colombiana de Psicologia, 27(1), 117-131. https:// doi.org/10.15446/rcp.v27n1.64621
Obradovic, I. (2017). Tabac, alcool et drogues illicites à l'adolescence: Évolution des consommations et enjeux. Revue des Politiques Social et Familiales, 125, 73-79.

Pérez-Gomez A., Lanziano C., Reyes-Rodríguez M. F., Mejía-Trujillo J., \& Cardozo-Macías F. (2018). Perfiles asociados al consumo de alcohol en adolescentes colombianos. Acta Colombiana de Psicología, 21(2), 258-269. https://doi.org/10.14718/ ACP.2018.21.2.12

Riquelme, M., García, O. F., \& Serra, E. (2018). Psychosocial maladjustment in adolescence: Parental socialization, self-esteem, and substance use. Anales de Psicología, 34(3), 536-544. https://doi.org/10.6018/analesps.34.3.315201.

Robinson, C. C., Mandleco, B., Olsen, S. F., \& Hart, C. H. (1995). Authoritative, authoritarian and permissive parenting practices: Development of a new measure. Psychological Reports, 77, 819830. https://doi.org/10.2466/pr0.1995.77.3.819

Roche, D. J. O., Bujarski, S., Green, R., Hartwell, E. E., Leventhal, A. M., \& Ray, L. A. (2019). Alcohol, tobacco, and marijuana consumption is associated with increased odds of same-day substance co-and tri-use. Drug and Alcohol Dependence, 200, 40-49. https://doi.org/10.1016/j.drugalcdep.2019.02.035

Sahed, I. (2016). Consommer la cigarette, le cannabis à l'adolescence: Quête identitaire et vulnérabilité dans le parcours de consommation. Drogues, Santé et Société, 14(2), 1-23. https:// doi.org/10.7202/1037730ar

Sanches, C., \& Gouveia-Perreira, M. (2010). Julgamentos de justiça em contexto escolar e comportamentos desviantes na adolescência. Análise Psicológica, 1(28), 71-84.

Sangawi, H., Adams, J., \& Reissland, N. (2016). The impact of parenting styles on children developmental outcome: The role of academic self-concept as a mediator. International Journal of Psychology, 53(5), 1-9. https://doi.org/10.1002/ijop.12380

Saud, L. F., \& Tonelotto, J. M. F. (2005). Comportamento social na escola: Diferenças entre gênero e séries. Psicologia Escolar e Educacional, 9(1), 47-57. https://doi.org/10.1590/S141385572005000100005

Saunders, J. B., Aasland, O. G., Babor, T. F., De La Fuente, J. R., \& Grant, M. (1993). Development of the alcohol use disorders identification teste (audit): Who collaborative project on early detection of persons with harmful alcohol consumption II. Addiction, 88, 791-804.

Skara, S., Pokhrel P., Weiner M. D., Sun, P., Dent, C. W., \& Sussman, S. (2008). Physical and relational aggression as predictors of drugs use: Gender differences among high school students. Addictive Behaviors, 33(12), 1507-1515. https://doi.org/10.1016/j. addbeh.2008.05.014

Tomé, G., Camacho, I., Matos, M. G., \& Simões, C. (2015). Influência da família e amigos no bem-estar e comportamentos de risco Modelo explicativo. Psicologia, Saúde \& Doenças, 16(1), 23-34. https://doi.org/10.15309/15psd160104

Tondowski, C., Bedendo, A., Zuquetto, A., Locatelli, D. P., Opaleye, E. S., \& Noto, A.R. (2015). Estilos parentais como fator de proteção ao consumo de tabaco entre adolescentes brasileiros. Caderno de Saúde Pública, 31(12), 2514-2522. https://doi. org/10.1590/0102-311X00168614

Weber, L. N. D. (2017). Relações entre práticas educativas parentais percebidas e a autoestima, sinais de depressão e ouso de substâncias por adolescentes. International Journal of Developmental and Educational Psychology, 2(1), 157-167.

Xue, Y., Zimmerman, M. A., \& Cunningham. R. (2009). Relationship between alcohol use and violent behavior among urban African American youths from adolescence to emerging adulthood: A longitudinal study. American Journal of Public Health, 99(11), 2041-2048. https://doi.org/10.2105/AJPH.2008.147827 